3. Santos JC, Passos GA. The high infectivity of SARS-CoV-2 B.1.1.7 is associated with increased interaction force between Spike-ACE2 caused by the viral N501Y mutation. bioRxiv 2020. doi: 10.1101/2020.12.29.424708.

4. Cheng MH, Krieger JM, Kaynak B, Arditi M, Bahar I. Impact of South African 501.V2 variant on SARS-CoV-2 spike infectivity and neutralization: a structure-based computational assessment. bioRxiv 2021. Doi: 10.1101/ 2021.01.10.426143.

5. Arif T. 501.V2 and B.1.1.7 variants of COVID-19: a new time-bomb in the making? Infect Control Hosp Epidemiol 2021. doi: 10.1017/ice.2020.1434.

6. Lei Y, Zhang J, Schiavon CR, et al. SARS-CoV-2 spike protein impairs endothelial function via downregulation of ACE2. bioRxiv 2020. doi: 10.1101/ 2020.12.04.409144.
7. Zhang L, Jackson CB, Mou H, et al. SARS-CoV-2 spike-protein D614G mutation increases virion spike density and infectivity. Nat Commun 2020; 11(1):6013.

8. Groves DC, Rowland-Jones SL, Angyal A. The D614G mutations in the SARS-CoV-2 spike protein: Implications for viral infectivity, disease severity and vaccine design. Biochem Biophys Res Commun 2020. doi: 10.1016/j.bbrc. 2020.10.109.

9. Korber B, Fischer WM, Gnanakaran S, et al. Tracking changes in SARS-CoV2 spike: evidence that D614G increases infectivity of the COVID-19 virus. Cell 2020;182:812-827.

\title{
Warnings regarding the potential coronavirus disease 2019 (COVID-19) transmission risk: Vaccination is not enough
}

\author{
Tianming Zhao MD candidate ${ }^{1, a}(\mathbb{0})$, Chengyang Hu MD ${ }^{1,2, a}$, Mubashir Ayaz Ahmed MD ${ }^{3}$ (1), Ce Cheng DO ${ }^{4}$,, Yue Chen ${ }^{5}$ (1) \\ and Chenyu Sun MD, MSc ${ }^{3}$ (i) \\ ${ }^{1}$ Department of Epidemiology and Health Statistics, School of Public Health, Anhui Medical University, Hefei, Anhui, China, ${ }^{2}$ Department of Humanistic Medicine, \\ School of Humanistic Medicine, Anhui Medical University, Hefei, Anhui, China, ${ }^{3}$ Internal Medicine, AMITA Health Saint Joseph Hospital Chicago, Chicago, Illinois, \\ United States, ${ }^{4}$ The University of Arizona College of Medicine at South Campus, Tucson, Arizona, United States and ${ }^{5}$ Department of Clinical Medicine, School of \\ the First Clinical Medicine, Anhui Medical University, Hefei, Anhui, China
}

To the Editor-Caused by the severe acute respiratory syndrome coronavirus-2 (SARS-CoV-2), the coronavirus disease 2019 (COVID-19) pandemic has continued to spread around the world, resulting in a global health emergency of inconceivable magnitude. ${ }^{1,2}$ Currently, several vaccines, including the PfizerBioNTech COVID-19 vaccine and the Moderna COVID-19 vaccine, have been authorized for emergency use to prevent COVID $-19 .^{3}$ A previous study indicated that the use of a vaccine in combination with measures that reduce contact between susceptible individuals and COVID-19 carriers will significantly decrease the per-day risk of infection as long as at least $50 \%$ of people receive it. ${ }^{4}$ In this article, these researchers also expressed their concern that potential vaccine defiance and abandoning other protection options may cause even worse results in COVID-19 prevention. ${ }^{4}$ In addition, due to the limited supply of COVID-19 vaccine in the United States, Centers for Disease Control and Prevention (CDC) recommends that initial supplies of SARS-CoV-2 vaccine be allocated to healthcare personnel and long-term care facility residents. $^{5}$ Considering the accessibility of vaccines in different regions and populations around the world, public health polices including keeping social distance and wearing face masks, are still of great importance, even though an effective vaccine has been introduced.

Furthermore, according to Morbidity and Mortality Weekly Report from the CDC, ${ }^{6}$ several issues still need to be explored: (1) No data assessing the efficacy of vaccine in prevention of asymptomatic SARS-CoV-2 infection are available; thus, the potential transmission risk of SARS-CoV-2 among asymptomatic

Author for correspondence: Chenyu Sun, E-mail: drsunchenyu@yeah.net

aAuthors of equal contribution.

Cite this article: Zhao T, et al. (2022). Warnings regarding the potential coronavirus disease 2019 (COVID-19) transmission risk: Vaccination is not enough. Infection Control \& Hospital Epidemiology, 43: 679-680, https://doi.org/10.1017/ice.2021.63 infected individuals cannot be ignored, even after vaccination. (2) Considering the time interval between the invention of the PfizerBioNTech SARS-CoV-2 vaccine and its emergency use authorization (EUA), the long-term effects of this vaccine (including adverse and protected effects) are still not entirely clear, and further surveillance is still necessary. (3) It takes $\sim 14$ days to obtain protection from infection after the first shot of Pfizer vaccine, ${ }^{7}$ and individuals may still be susceptible during the first few days to weeks after vaccination, whereas the general public may not fully understand this and may be less compliant with current nonpharmaceutical interventions (NPIs) immediately after receiving the vaccine.

In conclusion, uncertainties remain in the long-term effect of SARS-CoV-2 vaccines, and accessibility of vaccines is still limited. Strict public health policies aiming to reduce the spread of SARSCoV-2 are still warranted and should not be ignored.

Financial support. No financial support was provided relevant to this article.

Conflicts of interest. All authors report no conflicts of interest relevant to this article.

\section{References}

1. Twomey JD, Luo S, Dean AQ, et al. COVID-19 update: the race to therapeutic development. Drug Resist Updat 2020;53:100733.

2. Artese A, Svicher V, Costa G, et al. Current status of antivirals and druggable targets of SARS CoV-2 and other human pathogenic coronaviruses. Drug Resist Updat 2020;53:100721.

3. Different COVID-19 vaccines. Centers for Disease Control and Prevention website. https://www.cdc.gov/coronavirus/2019-ncov/vaccines/differentvaccines.html. Published 2020. Accessed February 8, 2021.

4. Abo SMC, Smith SR. Is a COVID-19 vaccine likely to make things worse? Vaccines (Basel) 2020;8(4):E761.

5. When vaccine is limited, who gets vaccinated first? Centers for Disease Control and Prevention website. https://www.cdc.gov/coronavirus/2019- 
ncov/vaccines/recommendations.html. Published 2020. Accessed February $8,2021$.

6. Oliver SE, Gargano JW, Marin M, et al. The Advisory Committee on Immunization Practices interim recommendation for use of PfizerBioNTech COVID-19 vaccine-United States, December 2020. Morb Mortal Wkly Rep 2020;69:1922-1924.
7. The Vaccines and Related Biological Products Advisory Committee meeting, December 10, 2020. FDA Briefing Document, Pfizer-BioNTech COVID-19 vaccine. US Food and Drug Administration website. https://www.fda.gov/ media/144245/download. Published 2020. Accessed February 8, 2021.

\title{
Impact of coronavirus disease 2019 (COVID-19) vaccination program on healthcare worker infections in an academic hospital
}

\author{
Erin Dunbar MD, MS, FAAP ${ }^{1}$ (1), Emily Godbout DO, $\mathrm{MPH}^{2}$, Rachel Pryor RN, MPH${ }^{3}$, Henry J. Rozycki MD ${ }^{4}$ and \\ Gonzalo Bearman MD, MPH${ }^{2}$ \\ ${ }^{1}$ Pediatric Emergency Department, Virginia Commonwealth University Health System, Richmond, Virginia, ${ }^{2}$ Department of Infectious Diseases, Virginia \\ Commonwealth University Health System, Richmond, Virginia, ${ }^{3}$ Hospital Infection Prevention Program, Virginia Commonwealth University Health System, \\ Richmond, Virginia and ${ }^{4}$ Department of Pediatrics, Division of Neonatal-Perinatal Medicine, Virginia Commonwealth University Health System, Richmond, Virginia
}

To the Editor-The third wave of the coronavirus disease 2019 (COVID-19) pandemic resulted in a significant rise in hospitalizations and healthcare worker (HCW) severe acute respiratory coronavirus virus 2 (SARS-CoV-2) infections. After implementing the SARS-CoV-2 vaccination program, despite rising COVID-19 hospitalizations, we promptly observed a decrease in HCW infections.

Our COVID-19 vaccination HCW program began on December 16, 2020, (Pfizer/BioNTech) and December 28, 2020 (Moderna). The COVID-19 cases were identified by nasal swab PCR testing of clinically symptomatic individuals. Six days after beginning employee immunizations, our HCW COVID-19 infection rate decreased by $25 \%$. After $60 \%$ of employees received the 1 st vaccine dose, the HCW COVID-19 rate decreased by $50 \%$ (Fig. 1). At 14-28 days and $>28$ days after their first vaccine dose, HCWs were less likely to have COVID-19 than those who did not receive the vaccine $(0.15 \%$ and $0.00 \%$ vs $0.59 \%, P=.0002$ and .0004 , respectively). Concurrently implemented SARS-CoV-2 transmission prevention strategies included the transition from cloth masks to level-3 masks for all employees, mandatory face shields for direct patient care, a restricted visitor policy, and physical space adjustments for improved social distancing.

The Pfizer/BioNTech clinical trial reported a vaccine efficacy of $95 \%$ at least 7 days after the second dose and protection as early as 12 days after administration of the first dose. ${ }^{2}$ The Moderna vaccine trial observed similar protection prior to the second dose. ${ }^{3}$ Our data are consistent with these studies and underscore the prompt benefits of vaccination for the prevention of COVID-19 in the healthcare system even prior to the completion of the second vaccine dose. Our additional infection control strategies are consistent with CDC recommendations ${ }^{4}$ and likely further optimized HCW safety.

We present a bundled infection prevention approach including vaccination for the prompt reduction of COVID-19 infection in HCWs. The impact of COVID-19 vaccination in HCWs was observed even prior to completion of the second dose. Wood et $\mathrm{al}^{5}$ suggest $12 \mathrm{key}$ strategies to promote vaccination, 2 of which are relevant here: increasing observability and countering anecdotal "bad reaction" with "good reaction" vaccine stories. ${ }^{5} \mathrm{We}$ share our vaccine story to encourage more vaccination-hesitant HCWs to receive immunizations and to receive them earlier. Reaching herd immunity through vaccination is a crucial next step in ending this pandemic.

Acknowledgments.

Financial support. No financial support was provided relevant to this article.

Conflicts of interest. All authors report no conflicts of interest relevant to this article.

\section{References}

1. Coronavirus key measures-COVID 19 in Virginia. Virginia Department of Health website. https://www.vdh.virginia.gov/coronavirus/key-measures/. Published 2020. Accessed February 8, 2021.
Author for correspondence: Erin Dunbar, E-mail: erin.dunbar@vcuhealth.org

Cite this article: Dunbar E, et al. (2022). Impact of coronavirus disease 2019 (COVID-

19) vaccination program on healthcare worker infections in an academic hospital. Infection

Control \& Hospital Epidemiology, 43: 680-681, https://doi.org/10.1017/ice.2021.62 